\title{
Preparation, Characterization, and Application of Nickel Oxide Nanoparticles in Glucose and Lactose Biosensors
}

\author{
A. M. Abdallah ${ }^{1}$, H. Basma ${ }^{1} \&$ R. Awad ${ }^{1}$ \\ ${ }^{1}$ Department of Physics, Beirut Arab University, Beirut, Lebanon \\ Correspondence: H. Basma, Department of Physics, Beirut Arab University, Beirut, Lebanon. E-mail: \\ h.basma@bau.edu.lb, hadibassma@gmail.com
}

Received: April 20, 2019

Accepted: May 5, 2019

Online Published: May 30, 2019

doi:10.5539/mas.v13n6p99

URL: https://doi.org/10.5539/mas.v13n6p99

\begin{abstract}
Nickel oxide ( $\mathrm{NiO})$ nanoparticles were synthesized using the co-precipitation method. The structural, morphological, optical and magnetic properties of $\mathrm{NiO}$ nanoparticles were investigated by $\mathrm{X}$-ray Powder Diffraction (XRD), Transmission Electron Microscopy (TEM), Fourier Transform Infrared Spectroscopy (FTIR), UV-vis Spectroscopy and Vibrating Sample Magnetometer (VSM). The influence of sonication time on the physical characteristics of $\mathrm{NiO}$ nanoparticles was illustrated. The interactions of $\mathrm{NiO}$ nanoparticles with both glucose and lactose have been0 studied using UV-vis Absorption Spectroscopy and Fluorescence Spectroscopy. XRD pattern reveals that $\mathrm{NiO}$ nanoparticles exhibit a face-centered-cubic lattice structure with a crystallite size of $22.77 \mathrm{~nm}$. The magnetic hysteresis demonstrates the ferromagnetic behavior of $\mathrm{NiO}$ nanoparticles at room temperature. The energy band gap increases, from $2.8 \mathrm{eV}$ to $3.1 \mathrm{eV}$, with increasing sonication time from 5 to 20 minutes. It is found that the interaction of lactose- $\mathrm{NiO}$ nanoparticles is stronger than that of glucose-NiO ones. The obtained results can be investigated in future biomedical applications, especially in non-enzymatic biosensors.
\end{abstract}

Keywords: Biosensors, Glucose, Lactose, Nanoparticles complexation, NiO nanoparticles, XRD

\section{Introduction}

Nickel oxide ( $\mathrm{NiO}$ ) nanoparticle besides of being a supreme transition metal oxide, it is one of the remarkable ptype semiconductors. The various features of $\mathrm{NiO}$ nanoparticles have stimulated different research topics. The quantum size, the highly specific surface area, the volume, and the macroscopic quantum tunnel effects have unveiled the unique magnetic, electronic, catalytic, chemical and optical properties of the $\mathrm{NiO}$ nanoparticles. Such properties encouraged their extensive application in superparamagnetic devices, electrochromic smart windows, photo-catalyst applications, electrochemical supercapacitors, photovoltaic devices and gas sensors (Rahal, Awad, Abdel Gaber, \& Bakeer, 2017) (Al Boukhary, Zeidan, Khalaf, \& Awad, 2019) (Meng et al., 2013) (Gao, Zeng, \& Miao, 2016). NiO nanoparticles are also magnetic nanoparticles with good electrochemical activity, which motivate their use in electrochemical biosensors (Koh \& Josephson, 2009) (Meng et al., 2013) (Nur et al., 2014).

Electrochemical biosensors have been studied for decades. Several studies employed the integration of different enzymes on variable electrodes in the development of glucose biosensors (Ang, Por, \& Yam, 2015). Lovic et al. (Lovic et al., 2018) studied glucose biosensors based on three enzymes: cysteine, glutaraldehyde and glucose oxidase attached to the gold electrode. Yang et al. (Yang, Gong, Miao, \& Xu, 2017) investigated glucose oxidase and horseradish peroxidase to prepare glucose biosensor depending on carbon nanotubes modified glassy carbon electrode. Liu et al. (Bian, Wang, Ye, \& Liu, 2017) deliberated glucose biosensors based on glucose oxidase attached to screen-printed electrodes with porous structure. Moreover, researcher groups utilized the enzymatic biosensors for lactose detection. Sharma et al. (Sharma et al., 2007) established a lactose biosensor based on immobilized lactase and galactose oxidase attached to the oxygen electrode of a dissolved oxygen analyzer. Ferreira et al. (Ferreira, Souza Jr, Trierweiler, Hitzmann, \& Foll, 2003) established an enzymatic lactose biosensor based on $\beta$-galactosidase and glucose oxidase. In addition, few studies employed enzymatic detection for both saccharides: glucose and lactose. Portaccio and Lepore (Portaccio \& M. Lepore, 2017) worked on Amperometric biosensor based on two enzymes: glucose oxidase and $\beta$-galactosidase stuck on graphite electrode for the detection of both saccharides. Moreover, some studies investigated the integration of metal oxides in glucose biosensors. Li et al. ( $\mathrm{Li}$ et al., 2016) worked on non-enzymatic glucose sensors depending on reduced graphene oxide supported binary catalyst of $\mathrm{NiO} / \mathrm{CuO}$ nanocomposites prepared on the glassy electrode. Nia et al. (Nia, 
Meng, Lorestani, Mahmoudian, \& Alias, 2015) prepared a non-enzymatic glucose sensor based on copper oxide/ polypyrrole nanofiber/ reduced graphene oxide nanocomposite. He et al. (He et al., 2018) proposed non-enzymatic glucose sensors based on cubic NiO hollow porous architecture electrode.

Yet, the enzymatic biosensors have drawbacks, as they encounter poor reproducibility, thermal and chemical instability that arises from the intrinsic nature of the enzyme. (Meng et al., 2013) (Li et al., 2016) (He et al., 2018).

Furthermore, the detection of glucose and lactose play a crucial role in the control of bioprocess, pharmaceutical analysis, food production, industrial, clinical and medical applications. For instance, the detection of glucose levels is utilized in various biotechnological processes and it is highly used by diabetic patients. Moreover, the monitoring of lactose level in milk identifies its quality and distinguishes the suitable items for lactose intolerant patients (Sharma et al., 2007) (Ferreira et al., 2003) (Portaccio \& M. Lepore, 2017) (Li et al., 2016) (He et al., 2018) (Zhang, Chen, Jiang, Xiang, \& Zhang, 2010).

This work is devoted towards the investigation of $\mathrm{NiO}$ nanoparticles' characteristics and their compatibility for further investigation in biomedical applications as well as the influence of sonication time on physical characteristics of $\mathrm{NiO}$ nanoparticles and $\mathrm{NiO}$-glucose-lactose interactions.

\section{Experimental Techniques}

\subsection{Nickel oxide Nanoparticles'Synthesis}

Nickel oxide $(\mathrm{NiO})$ nanoparticles were prepared using the co-precipitation method. $1 \mathrm{M}$ of Nickel chloride hexahydrate $\mathrm{NiCl}_{2} \cdot 6 \mathrm{H}_{2} \mathrm{O}$ was titrated with $4 \mathrm{M}$ alkali sodium hydroxide $(\mathrm{NaOH})$ solution where its $\mathrm{pH}$ was adjusted to 13 . The obtained green solution was heated at $60^{\circ} \mathrm{C}$ for 2 hours followed by sonication for 3 minutes. After sonication, the viscous sample was washed several times by distilled water until its $\mathrm{pH}$ drops to 7 . Then, the green precipitate was dried at $100^{\circ} \mathrm{C}$ for 16 hours followed by manual grinding. The obtained fine green powders were calcinated at $550^{\circ} \mathrm{C}$ for 6 hours. Finally, the resultant black powders were crushed using high-speed ball milling for 20 minutes at a rate of $400 \mathrm{rpm}$ and a 5:1 weight ratio of ball to powder was used.

\subsection{Nanoparticles Characterization}

X-ray diffraction (XRD) was resorted to determine the structural properties using Bruker AXS D8 advance powder diffractometer with $\mathrm{Cu}-\mathrm{K}_{\alpha}$ radiation $(\lambda=1.54056 \AA)$ in the range of $10^{\circ} \leq 2 \theta \leq 80^{\circ}$, and Transmission electron microscopy (TEM) was utilized to find the morphological ones, using JEM $100 \mathrm{CX}$, operated at $80 \mathrm{kV}$. Then, the Fourier Transform Infrared Spectroscopy (FTIR) was applied to examine the basis of the functional groups and the vibrational modes of different elements in the sample, using Nicolet iS5 FTIR spectrometer. Finally, the Ultraviolet-visible near infrared Spectrophotometer (NIR), V-670, and the Vibrating Sample Magnetometer (VSM), Lakeshoren7410, were used to investigate the optical and the magnetic properties at room temperature, respectively.

\subsection{Spectral Measurements}

The interactions of the $\mathrm{NiO}$ nanoparticles with the monosaccharides glucose and disaccharides lactose were studied in phosphate buffer medium of concentration $0.1 \mathrm{M}$ and $\mathrm{pH}$ 6.5. These conditions are highly recommended for the low noise level in biosensor's response ((Ang et al., 2015)(Sharma et al., 2007). The concentration of glucose and lactose is fixed in all the experiments at $1 \times 10^{-4} \mathrm{M}$. However, the concentration of $\mathrm{NiO}$ nanoparticles rises from $1 \times 10^{-4} M$ till $10 \times 10^{-4} M$. These interactions were analyzed by UV-vis spectroscopy and Fluorescence spectroscopy, FP-8300. Accordingly, the binding constant was determined from the absorption and emission spectra as well.

\section{Results and Discussions}

\subsection{Nanoparticles' Characterization}

Figure 1 demonstrates the $\mathrm{X}$-ray diffraction patterns for the $\mathrm{NiO}$ nanoparticles. Five significant diffraction peaks were detected at approximately $2 \theta=37.3^{\circ}, 44.4^{\circ}, 62.83^{\circ}, 76.3^{\circ}$ and $79.3^{\circ}$ referring to (111), (200), (220), (311) and (222) crystalline planes. The XRD spectrum indicates the face-centered-cubic lattice structure as well as the high degree of crystallinity of the synthesized NiO nanoparticles. The obtained spectrum is comparable to that obtained by Rahal et al. (Rahal et al., 2017). The lattice constant a the average crystallite size (D) and the X-ray density $\rho$ of $\mathrm{NiO}$ are calculated from the following formulas (Al Boukhari, 2019) (Waseda, Matsubara, \& Shinoda, 2011):

$$
\begin{gathered}
\mathrm{a}=\mathrm{d}_{\mathrm{hkl}} \sqrt{\mathrm{h}^{2}+\mathrm{k}^{2}+\mathrm{l}^{2}} \\
\mathrm{D}=k \lambda / \beta \cos \theta \\
\rho=Z M / N_{A} a^{3}
\end{gathered}
$$


$(\mathrm{h}, \mathrm{k}, \mathrm{l})$ are the Miller indices, $\mathrm{d}$ is the spacing between adjacent (hkl) lattice planes, $\beta=$ FWHM (full width at half maximum), $\theta$ is glancing angle evaluated from the diffraction angle $(2 \theta)$ values corresponding to maximum intensity peak in XRD pattern (2 00$), \mathrm{k}$ is an empirical constant equal to $0.9, \mathrm{Z}$ is the number of lattice points per unit cell, $\mathrm{M}$ is the molecular weight and $\mathrm{N}_{\mathrm{A}}$ is the Avogadro's number. The evaluated values are $4.189 \mathrm{~nm}$ for (111) lattice plane, $22.77 \mathrm{~nm}$ and $6.747 \times 10^{3}\left(\mathrm{Kg} / \mathrm{m}^{3}\right)$, respectively. These values are in reasonable agreement with that reported in the literature (Rahal et al., 2017) (Rifaya, Theivasanthi, \& Alagar, 2012). The specific surface area (SSA) is the ratio of surface area $\left(\mathrm{SA}=1,628.83 \mathrm{~nm}^{2}\right)$ to the mass of the nanoparticles (Rifaya et al., 2012):

$$
\mathrm{SSA}=S A / M=S A / \rho \times V
$$

where $\mathrm{V}=6181.41 \mathrm{~nm}^{3}$ is the particle volume. SSA is equal to $39.055 \times 10^{3} \mathrm{~m}^{2} \mathrm{Kg}^{-1}$. It is noticed that $\mathrm{NiO}$ nanoparticles synthesized by co-precipitation method - present work- have a lower specific surface area to that synthesized by chemical capping with a difference of $35 \times 10^{3} \mathrm{~m}^{2} / \mathrm{Kg}$ (Rifaya et al., 2012). However, they have a higher specific surface area to that synthesized by anodic arc plasma with a difference of $6 \mathrm{~m}^{2} / \mathrm{Kg}$ (Qiao, Wei, Yang, Zhu, \& Yan, 2009). In addition, the NiO nanoparticles have a high specific surface area that boosts their catalytic uses (Kolhatkar, Jamison, Litvinov, Willson, \& Lee, 2013).

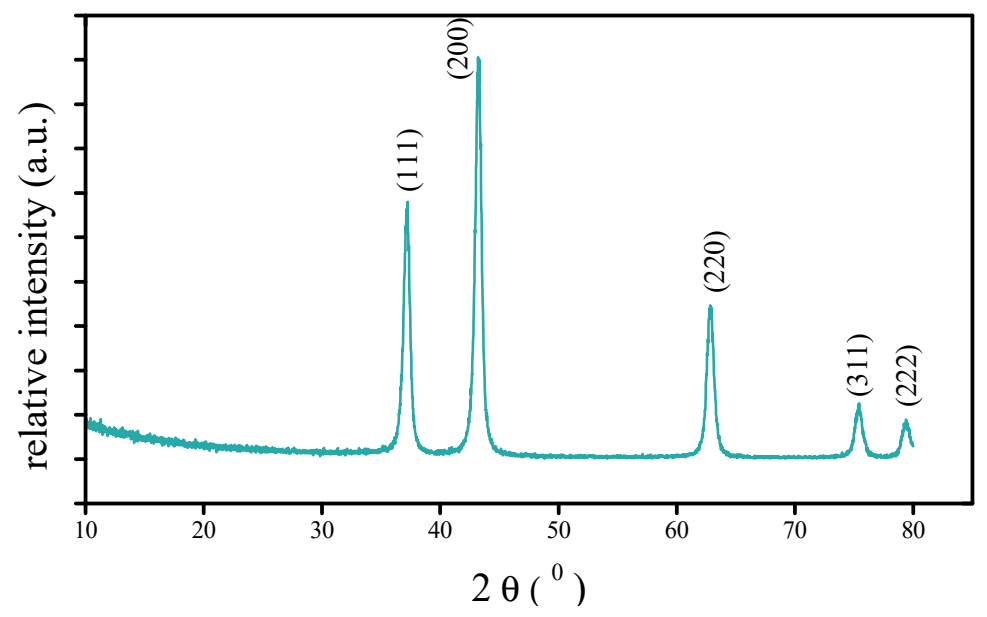

Figure 1. XRD pattern of $\mathrm{NiO}$ nanoparticles

Figure 2 reveals the size, shape, and morphology of the prepared $\mathrm{NiO}$ nanoparticles investigated by TEM. The average crystallite size is found to be $26.59 \mathrm{~nm}$, which is slightly greater than that obtained from XRD analysis by $7.74 \%$. Moreover, the figure confirms the spherical shape of $\mathrm{NiO}$ nanoparticles with weak agglomeration. The spherical shape offers a good contact area, confirming the calculated value of SSA, which is recommended for biosensing applications (Kolhatkar et al., 2013).

The crystallinity index $\left(I_{\text {crys }}\right)$ assigns the mono-crystallinity or poly-crystallinity of the nanoparticles if its value is approaching or exceeding one, respectively. It is evaluated using the following equation (Rifaya et al., 2012):

$$
I_{\text {crys }}=D(T E M) / D(X R D)
$$

The crystallinity index is equal to 1.167 , suggesting the mono-crystallinity of $\mathrm{NiO}$ nanoparticles. 


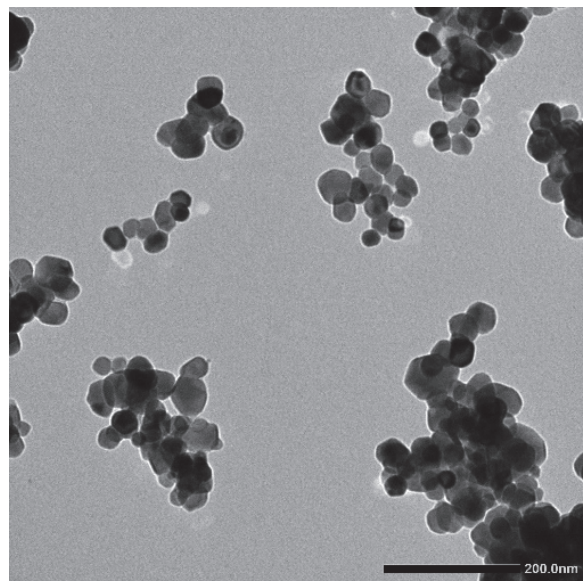

Figure 2. The TEM image of $\mathrm{NiO}$ nanoparticles

Figure 3 represents the FTIR spectrum of the $\mathrm{NiO}$ nanoparticles. The spectrum demonstrates a significant peak at $415 \mathrm{~cm}^{-1}$ which is related to the vibrational mode of Ni-O bond. This assures the formation of $\mathrm{NiO}$ nanoparticles. In addition, less significant peaks were detected at $1640 \mathrm{~cm}^{-1}$ and $3440 \mathrm{~cm}^{-1}$. These peaks refer to H-O-H bending vibrations mode and O-H stretching vibrations bond, respectively (Rahal et al., 2017) (Al Boukhari, 2019). The appearance of such peaks suggests a humidity percentage in the sample. This may be due to the increased contact of the sample with air or due to the initial preparation conditions (Rahal et al., 2017).

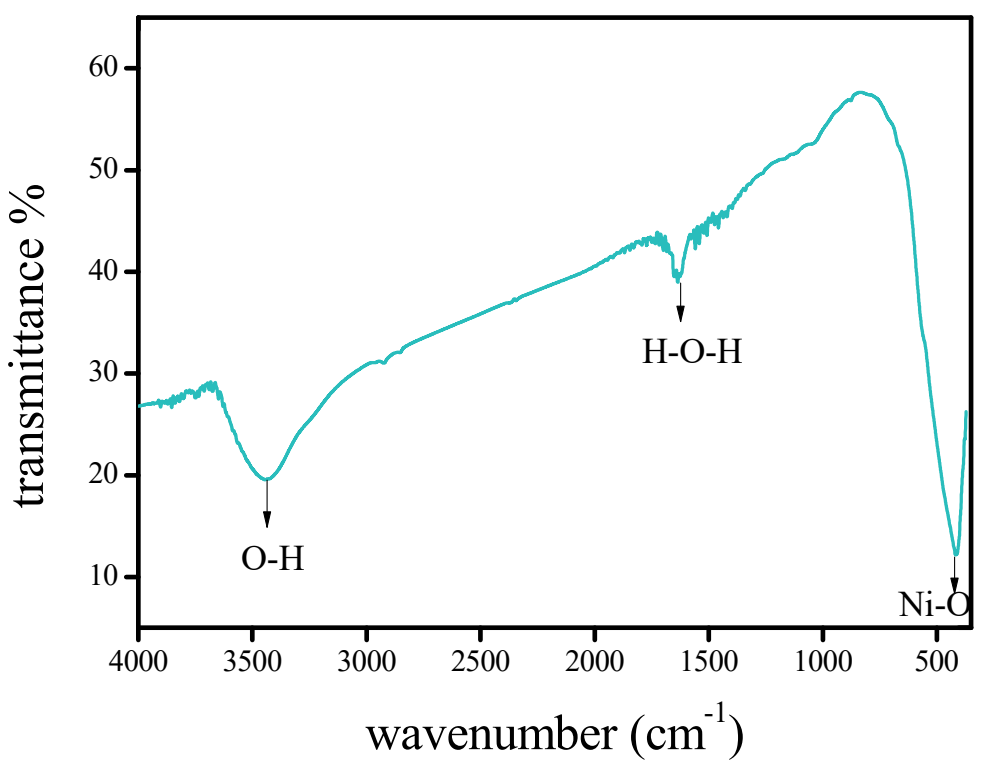

Figure 3. FTIR Spectra of calcinated $\mathrm{NiO}$

Figure 4 reveals the optical absorption spectra of $\mathrm{NiO}$ nanoparticles dissolved in absolute ethanol at different ultrasonic dispersion time. As seen, with increasing sonication time the absorption edge is blue shifted from 336 to $319 \mathrm{~nm}$ as well as the absorbance intensity increases. This can be attributed to the fact that the sonication decreases the size of $\mathrm{NiO}$ nanoparticles. To assure this result the degradation rate was calculated using equation (Khademolhoseini \& Talebi, 2015):

$$
\text { degradation rate }(\%)=\frac{A_{0}-A}{A_{0}} \times 100
$$

where $A_{0}$ and $A$ are the absorbance value of the solution at $t_{0}=5 \mathrm{~min}$ and $t$ respectively. The values are traced in the inset of figure 4 that exhibits a linear relation between the degradation rate and the sonication time. 
The empirical equation describing this relation is found to be:

$$
\text { degradaton rate }=-3.7327 t-2.3123
$$

It is noticed that the degradation rate increases negatively with sonication time. This indicates that the NiO nanoparticles break down into smaller nanocrystals with rising sonication time (Kashani-Motlagh, Rahimi, \& Kachousangi, 2009). This is in complete agreement with the absorption spectra results.

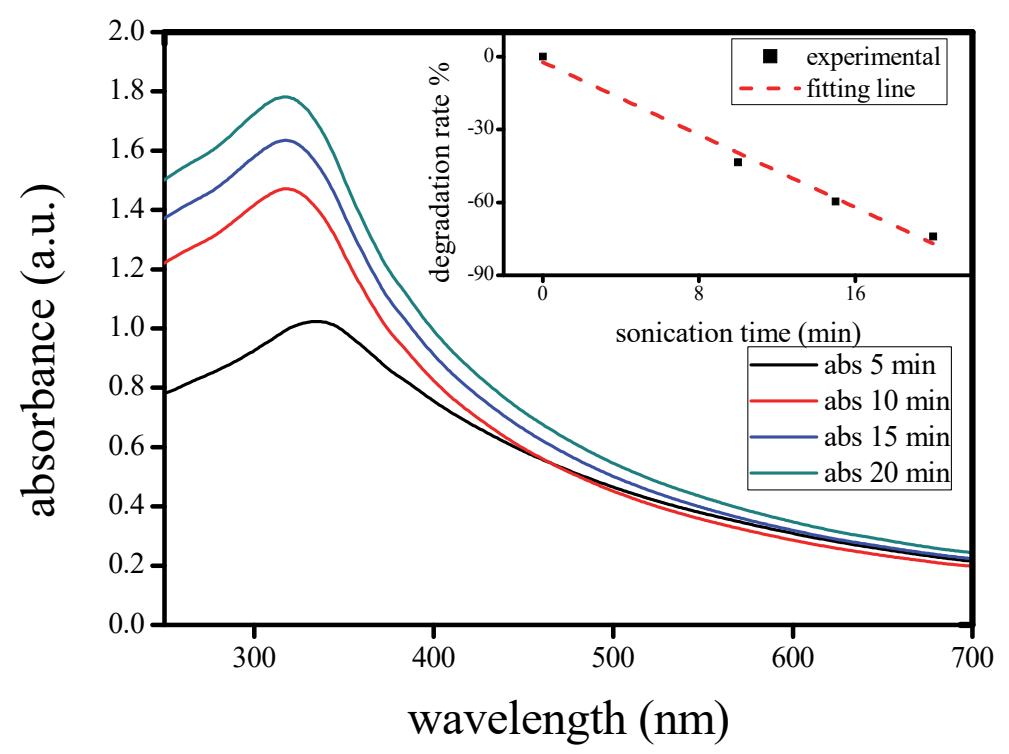

Figure 4. UV-vis absorption spectra of pure $\mathrm{NiO}$ at different sonication time

The energy bandgaps $\left(\mathrm{E}_{\mathrm{g}}\right)$ of the samples were calculated by the use of equation 8 as suggested by Davis and Mott (Al Boukhary, Zeidan, Khalaf, \& Awad, 2019):

$$
(\alpha h v)^{1 / n}=A\left(h v-E_{g}\right)
$$

where $\mathrm{A}$ is a constant, $h v$ is the photon energy and the exponent $\mathrm{n}$ depends on the type of the transition. It corresponds to $1 / 2,2,3 / 2$, or 3 for direct allowed, indirect allowed, direct forbidden or indirect forbidden transitions, respectively (Hassanien \& Akl, 2016). In the present work, the energy band gap is evaluated according to the direct transition, as it is the $\mathrm{x}$-intercept of the extrapolated linear portions of the curves, as shown in figure 5. It is inferred that the energy band gap increases with sonication time. This may be due to the crystallite degradation with more sonication (Kashani-Motlagh et al., 2009). Similar conclusion was achieved by Farhat et al that confirmed that the energy gap and the crystallite size are inversely proportional (Farhat, Rekaby, \& Awad, 2018).

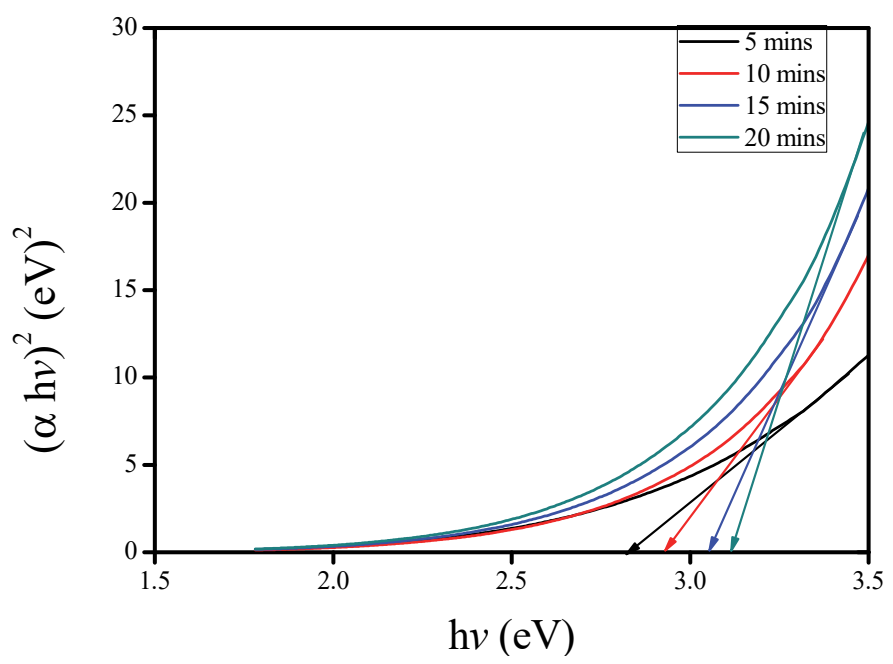

Figure 5. Energy gap at different sonication time 
The Urbach energy $E_{u}$ or the absorption band tail is the result of the extended localized states in the band gap. It arises from the exponential tail of the absorption coefficient $(\alpha)$ and it is given by the empirical relation (Hassanien \& Akl, 2016):

$$
\alpha(v)=\beta \exp .\left(h v / E_{u}\right)
$$

where $\beta$ is a constant. The Urbach energy $E_{u}$ can be estimated by plotting the logarithm of the absorption coefficient, $\ln (\alpha)$ as a function of the photon energy $h v$, as shown in figure 6 .

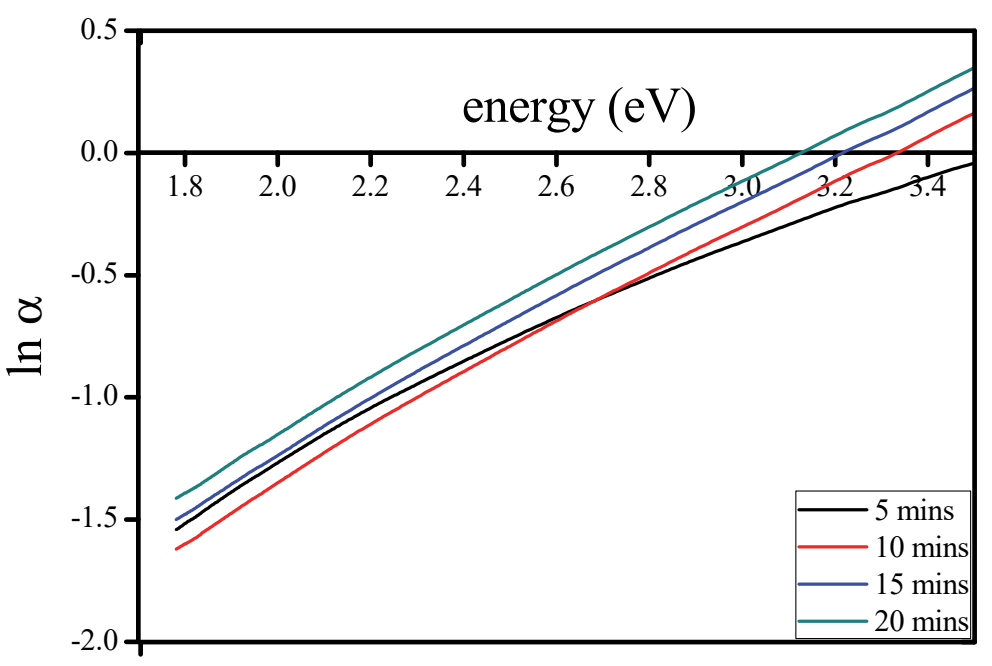

Figure 6. Urbach energy at different sonication time

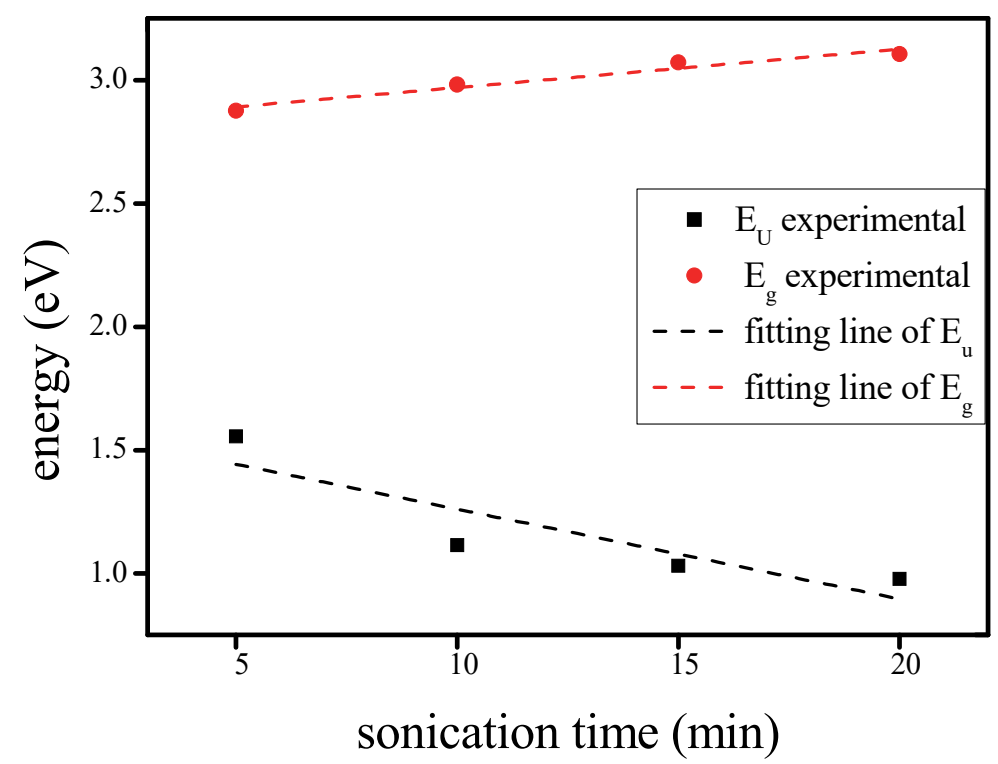

Figure 7. The relation between $\mathrm{E}_{\mathrm{g}}$ and $\mathrm{E}_{\mathrm{u}}$ with sonication time 


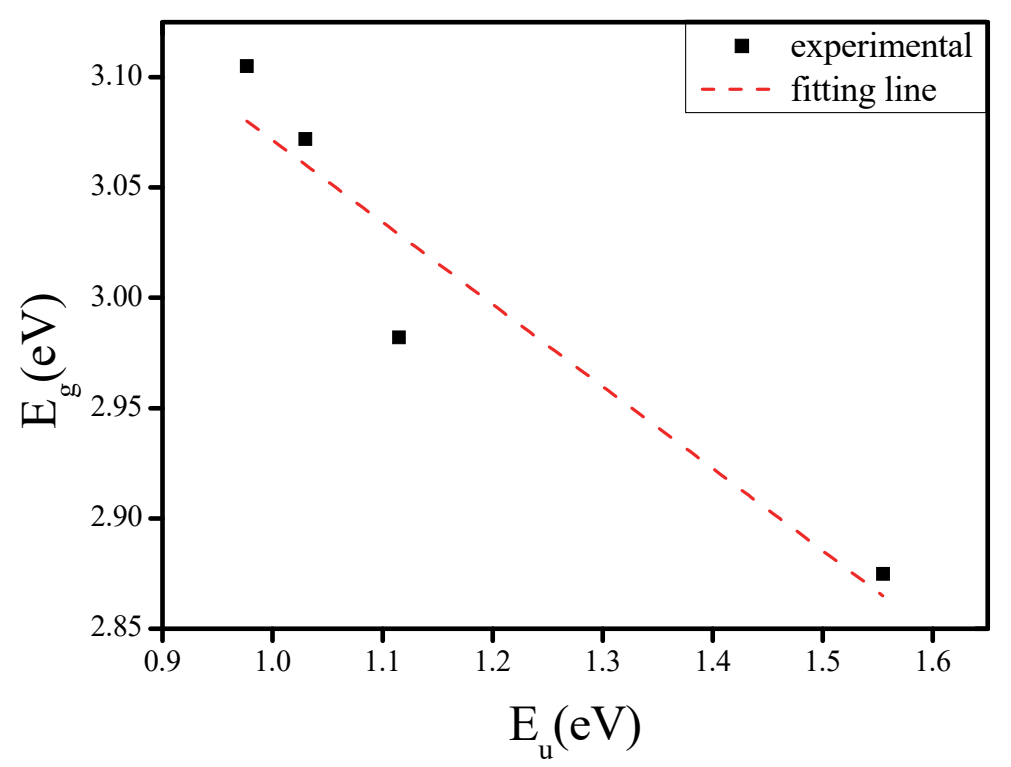

Figure 8. The relation between $\mathrm{E}_{\mathrm{g}}$ and $\mathrm{E}_{\mathrm{u}}$

The calculated values of $E_{g}$ and $E_{u}$ of the samples are traced in figure 7 versus sonication time. It is clearly observed that the Urbach energy, contrary to the energy gap, decreases as sonication time increases. Thus suggesting a relation between the energy gap and the Urbach energy. This relation is assured in figure 8, upon plotting the $\mathrm{E}_{\mathrm{g}}$ versus $\mathrm{E}_{\mathrm{u}}$. The following empirical formula is obtained with a correlation value of $R^{2}=0.9049$ :

$$
E_{g}=-0.3723 E_{u}+3.4438
$$

A similar linear fitting equation was obtained with different parameters by Hassanien et al [28] who studied the chalcogenide cadmium sulphoselenide $\mathrm{CdS}_{\mathrm{x}} \mathrm{Se}_{1-\mathrm{x}}$ thin films.

Figure 9 displays the $\mathrm{M}-\mathrm{H}$ loop of $\mathrm{NiO}$ nanoparticles. As seen, a ferromagnetic behavior is attained at room temperature. Such observation permits its use in biosensors applications (Koh \& Josephson, 2009). The remnant magnetization $\left(\mathrm{M}_{\mathrm{r}}\right)$ and the coercive field $\left(\mathrm{H}_{\mathrm{c}}\right)$ were obtained from the $\mathrm{M}-\mathrm{H}$ loop with values of $46.665 \times 10^{-3}$ $\mathrm{emu} / \mathrm{g}$ and $123.80 \mathrm{Oe}$, respectively. The saturation magnetization $\mathrm{M}_{\mathrm{s}}$ is estimated from the extrapolation of the curve obtained from the magnetization versus the reciprocal of the applied magnetic field. It is represented in the inset of figure 9 and it is found to be $0.926 \mathrm{emu} / \mathrm{g}$. The $\mathrm{M}_{\mathrm{s}}$ of the prepared $\mathrm{NiO}$ nanoparticles has a higher value compared to other ferromagnetic nanoparticles listed in table 1 which is a good characteristic that serves in the manufacture of several biomedical applications especially biosensors one (Colombo et al., 2012). The squareness $\mathrm{S}$ can be calculated from the following equation:

$$
S=M_{r} / M_{S}
$$

Its value is $65.64 \times 10^{-3}$ that reveals the magneto-static interaction between the particles (Al Boukhari, 2019). As a result, the ferromagnetic behavior of the $\mathrm{NiO}$ nanoparticles with high magnetic saturation recommend their use in glucose and lactose biosensors. 


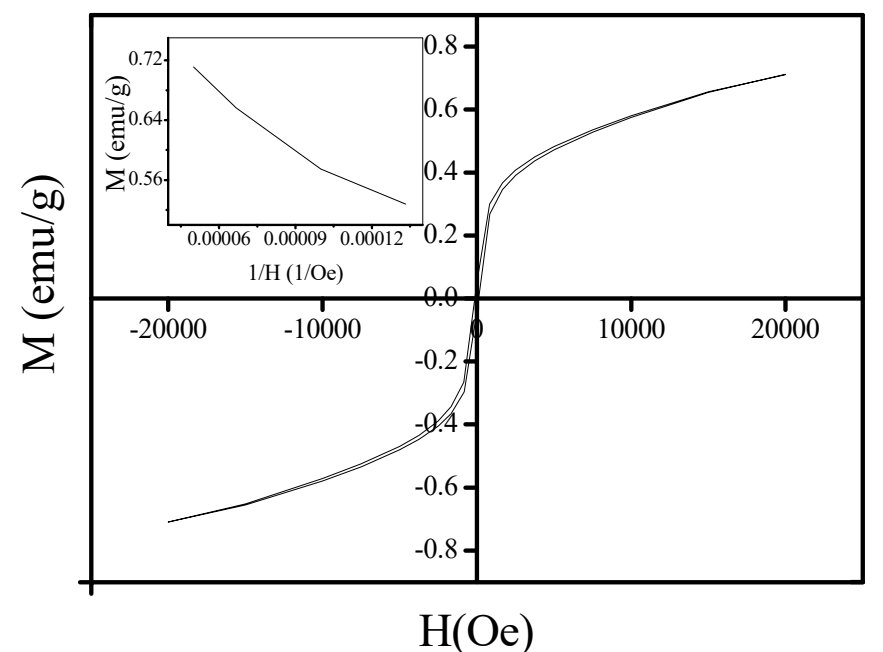

Figure 9. $\mathrm{M}-\mathrm{H}$ loop for $\mathrm{NiO}$ nanoparticles at room temperature

Table 1. The saturation magnetization for different nanoparticles

\begin{tabular}{llll}
\hline Materials & Size (nm) & Saturation magnetization (emu/g) & Reference \\
\hline $\mathrm{NiO}$ & 32.65 & 0.71087 & Present work \\
$\mathrm{Zn} 0$ & 25 & 0.0054 & (Gao et al, 2009) \\
$\mathrm{CeO}_{2}$ & 15 & $1.87 \times 10^{-3}$ & (Sundaresan \& Rao, 2009) \\
$\mathrm{Al}_{2} \mathrm{O}_{3}$ & 4 & $4.79 \times 10^{-3}$ & (Sundaresan \& Rao, 2009) \\
\hline
\end{tabular}

\subsection{Interaction between NiO Nanoparticles and the Carbohydrates}

Figures 10 (a) and (b) reveal the UV-vis absorption spectra of $\mathrm{NiO}$ nanoparticles with glucose and lactose dissolved in phosphate buffer solution, respectively. It is inferred that the increase of $\mathrm{NiO}$ nanoparticles concentration leads to a significant enhancement in the absorbance and a shift in the peak wavelength. Such behavior indicates the formation of glucose- $\mathrm{NiO}$ and lactose-NiO complexes as well (Hazra, Chakrabarty, Chakraborty, \& Sarkar, 2004). The spectrophotometric data is investigated to find the binding constant from the Benesi-Hildebrand equation (Yang, Liu, Mu, \& Guo, 2000) (Khouri \& Buss, 2011):

$$
\frac{1}{\Delta A_{i}}=\frac{1}{K \Delta A} \frac{1}{[N i O]_{i}}+\frac{1}{\Delta A}
$$

where $\mathrm{K}$ is the binding constant, $\Delta A_{i}=A_{i}-A_{0}$ for which $A_{i}$ and $A_{0}$ are the absorbance in the presence and absence of $\mathrm{NiO}$ nanoparticles respectively, $\Delta A=\varepsilon_{\infty}-\varepsilon_{0} /[$ glucose $]$ for which $\varepsilon_{\infty}$ and $\varepsilon_{0}$ represent the molar absorption constants of the glucose/lactose and glucose- $\mathrm{NiO} /$ lactose-NiO complex, respectively and $[\mathrm{NiO}]_{i}$ represents the different concentrations of $\mathrm{NiO}$ nanoparticles. Figures 11 (a) and (b) display the variation of $1 / \Delta A_{i}$ with the reciprocal of the concentration. A straight line is obtained from which $\mathrm{K}$ is evaluated as the ratio of the intercept to the slope. The binding constant values are found to be $1.7 \times 10^{2} \mathrm{M}^{-1}$ and $3.29 \times 10^{2} \mathrm{M}^{-1}$ for glucose-NiO and lactose-NiO interactions, respectively. El-Kemary et al. (El-Kemary, Nagy, \& El-Mehasseb, 2013) obtained similar observations in his study on the interactions of $\mathrm{NiO}$ nanoparticles with glucose. It is noted that the binding constant of lactose- $\mathrm{NiO}$ interaction is greater than that of glucose- $\mathrm{NiO}$ one. 

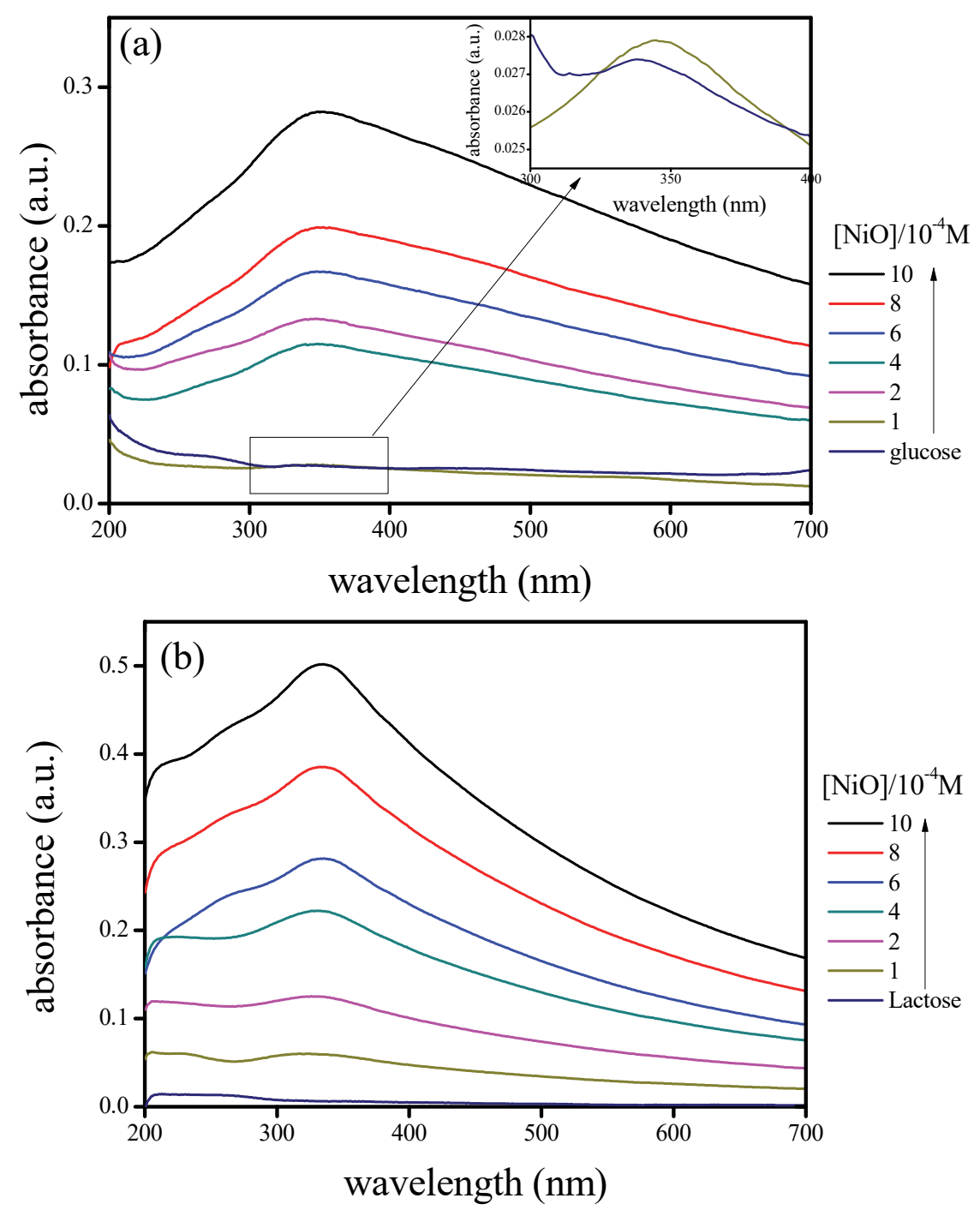

Figure 10. Absorption spectra for (a) glucose and (b) lactose with different concentrations of $\mathrm{NiO}$

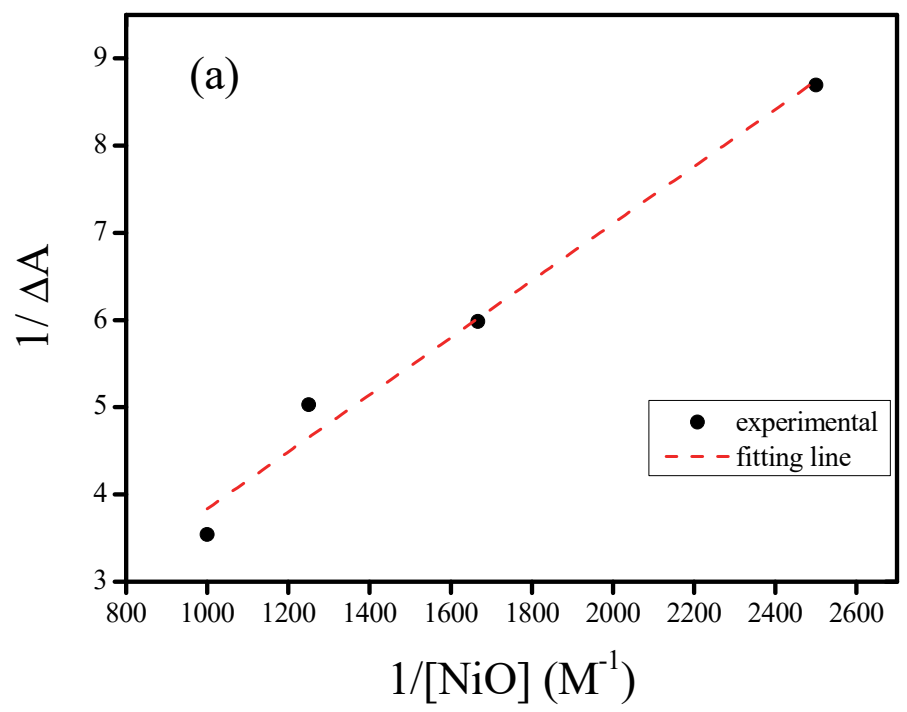




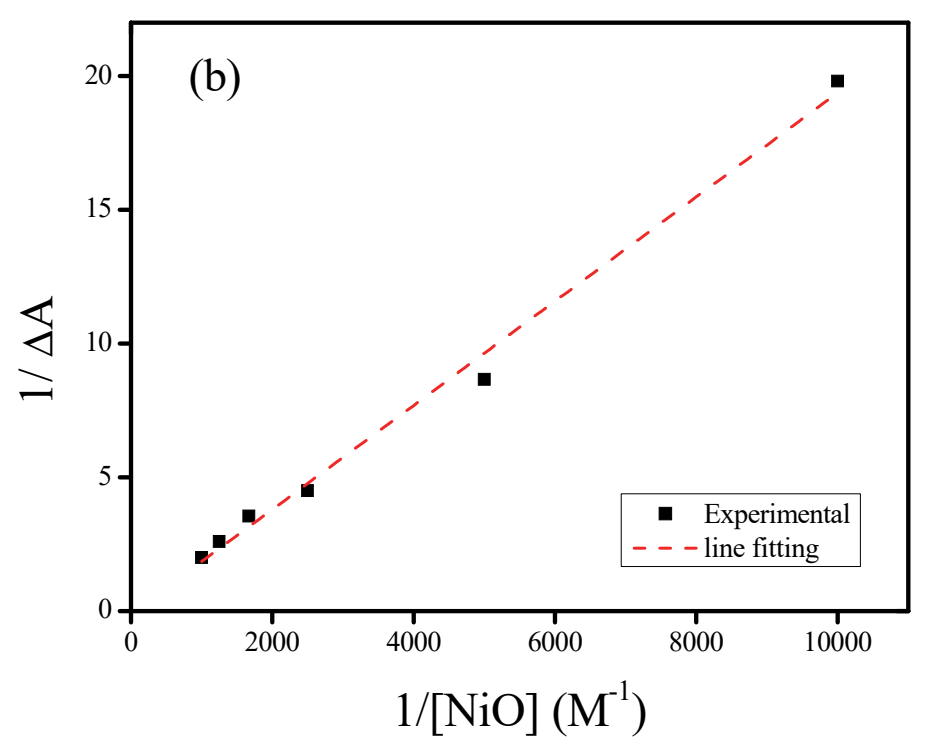

Figure 11. Benesi-Hildebrand plot for (a) glucose-NiO and (b) lactose-NiO interactions

Figures 12 (a) and (b) show the fluorescence spectra for the complexation between glucose-NiO and Lactose-NiO nanoparticles, respectively. Upon addition of $\mathrm{NiO}$ nanoparticles, the fluorescence intensity in both complexations increased from 107 to 157 au for glucose-NiO complex, and from 94 to 207 au for lactose-NiO complex. Such enhancement in the fluorescence intensities signifies the formation of inclusion complex with $\mathrm{NiO}$ nanoparticles (Hazra et al., 2004) (Yang et al., 2000). Besides the enhancement of the peaks, the peaks' wavelengths deviated for glucose- $\mathrm{NiO}$ and lactose-NiO interactions. However, the deviation for lactose-NiO interactions was more intensified than that of glucose-NiO ones. These deviations are attributed to the complexation process (Hazra et al., 2004) (Jana, Ghosh, \& Chattopadhyay, 2013) (Manzoori \& Amjadi, 2003) (Barragan, Fornue, Ortega, Gonzalez, \& Quinones, 2016). To assure the inclusion complex formation, the binding constant was estimated from the double reciprocal formula:

$$
\frac{1}{I-I_{0}}=\frac{1}{\left(I_{1}-I_{0}\right) K[N i O]}+\frac{1}{I_{1}-I_{O}}
$$

where $\mathrm{I}_{0}$ represents the fluorescence intensity of glucose/ lactose, $\mathrm{I}_{1}$ represents the fluorescence intensity of the inclusion complex and I represent the obtained fluorescence intensity of glucose-NiO and lactose-NiO complex at their maxima.

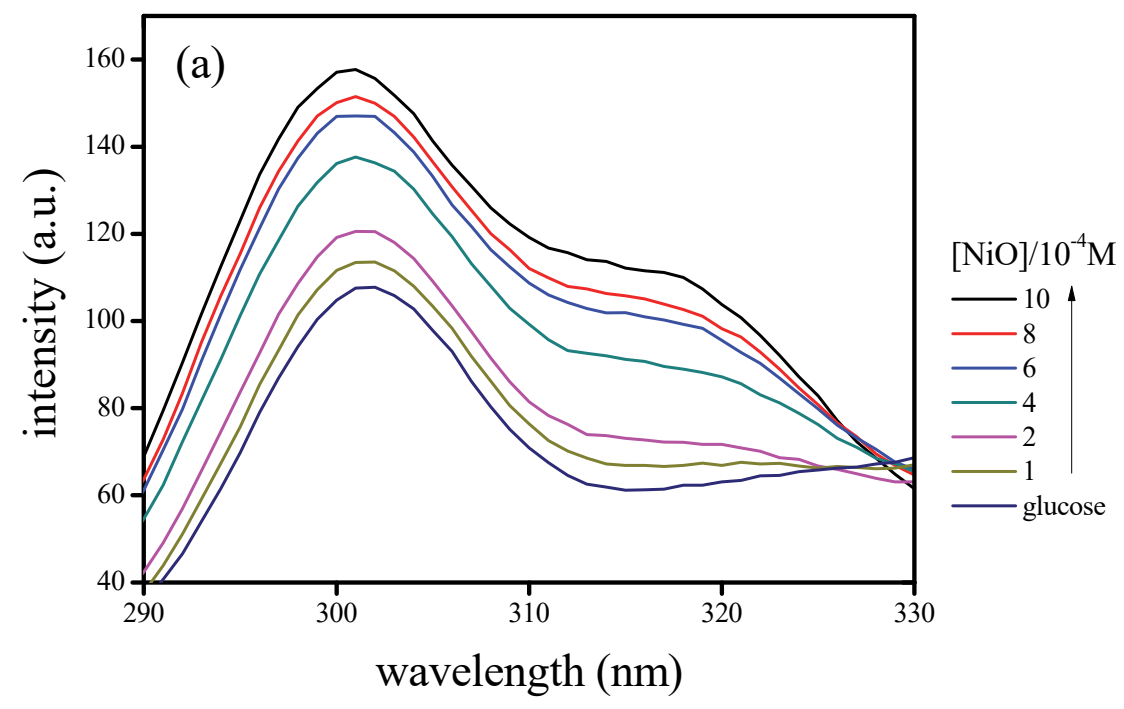




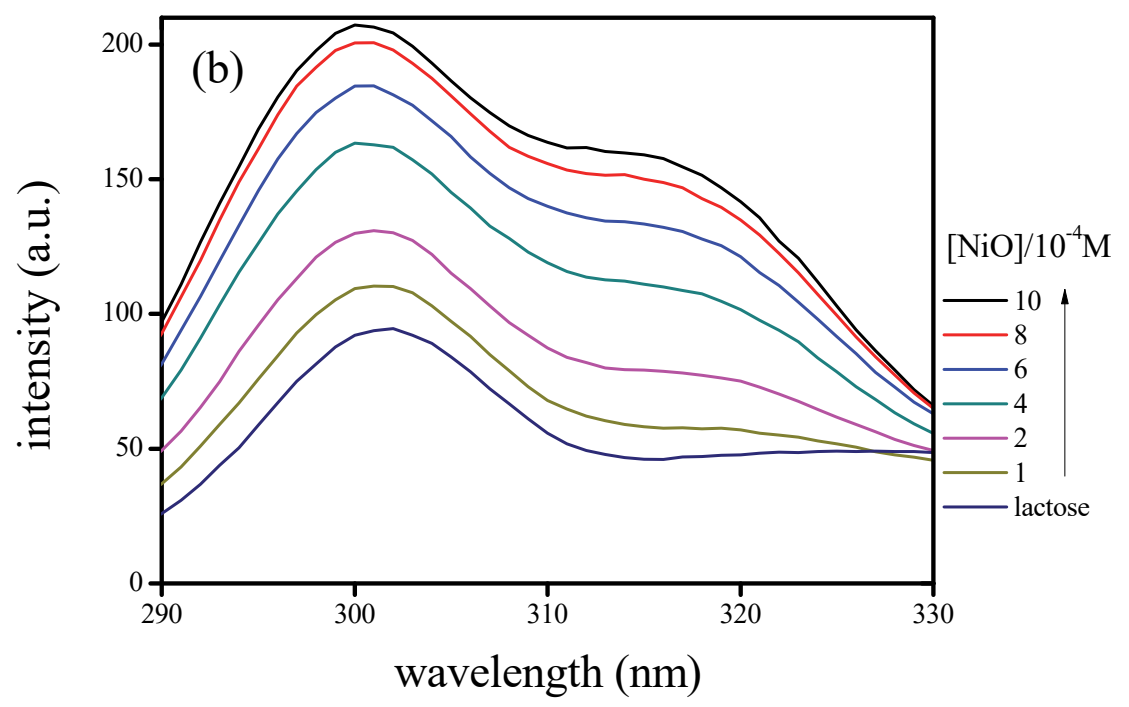

Figure 12. Fluorescence spectra for (a) glucose and (b) lactose with different concentrations of $\mathrm{NiO}$

Figure 13 represents the plot of $1 / I-I_{0}$ for (a) glucose-NiO interaction and (b) lactose-NiO interaction versus the reciprocal of $[\mathrm{NiO}]$. Straight lines were obtained indicating 1:1 stoichiometry in glucose-NiO and lactose-NiO inclusion complex (Hazra et al., 2004) (Manzoori \& Amjadi, 2003) (Barragan et al., 2016). Consequently, the binding constant was found to be $1.4 \times 10^{2} \mathrm{M}^{-1}$ and $2 \times 10^{2} \mathrm{M}^{-1}$ for glucose-NiO and lactose-NiO inclusion complex, respectively. These values are in reasonable agreement with that calculated from the Benesi-Hildebrand data. Moreover, it is noted that the binding constant of lactose-NiO inclusion complex is higher than that of glucose- $\mathrm{NiO}$ one. Thus, the results propose the use of $\mathrm{NiO}$ nanoparticles in non-enzymatic lactose biosensors. The Gibbs energy $\Delta G^{0}$ of glucose-NiO and Lactose-NiO interaction were calculated from the following thermodynamic equation (Khouri \& Buss, 2011):

$$
\Delta G^{0}=-2.303 R T \log K
$$

where $\mathrm{R}$ is the ideal gas constant and $\mathrm{T}$ is the absolute temperature. The estimated values of Gibbs energy are $12.245 \mathrm{KJ} / \mathrm{mol}$ and $-13.12 \mathrm{KJ} / \mathrm{mol}$ for glucose- $\mathrm{NiO}$ and lactose-NiO interactions, respectively. Such values signify the stable and spontaneous interactions between glucose and lactose with NiO nanoparticles (Khouri \& Buss, 2011). Similar results were attained by Barragán et al. (Barragan et al., 2016) in his study on the complexation of 2,5$\operatorname{Bis}(5$-tert-butyl-benzoxazol-2yl)-thiophene with native $\beta$-cyclodextrin and $\beta \mathrm{CD}$-monochlorotriazinyl.

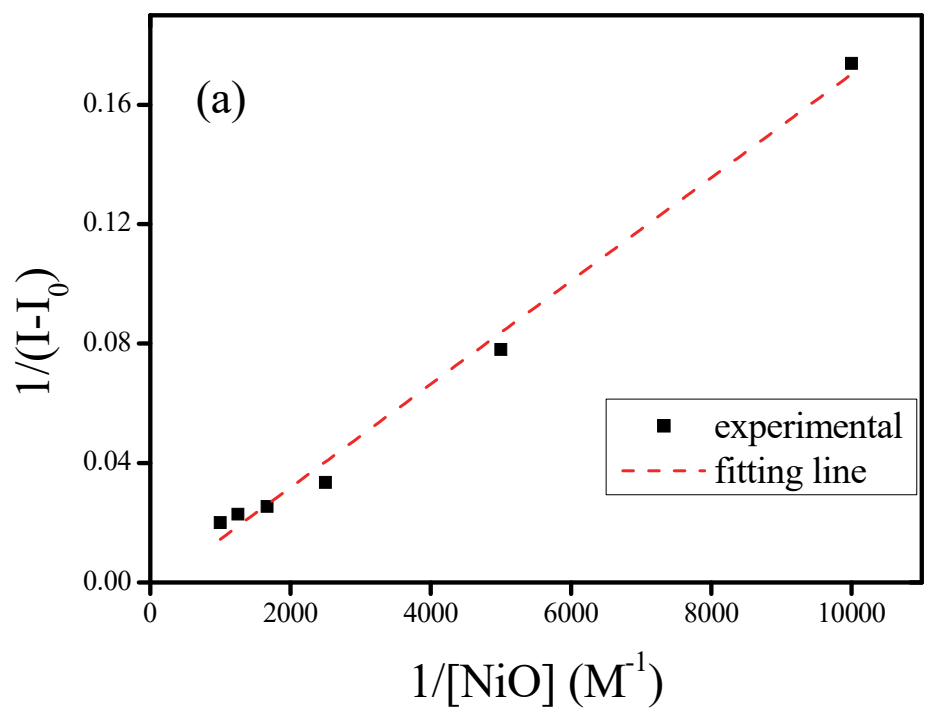




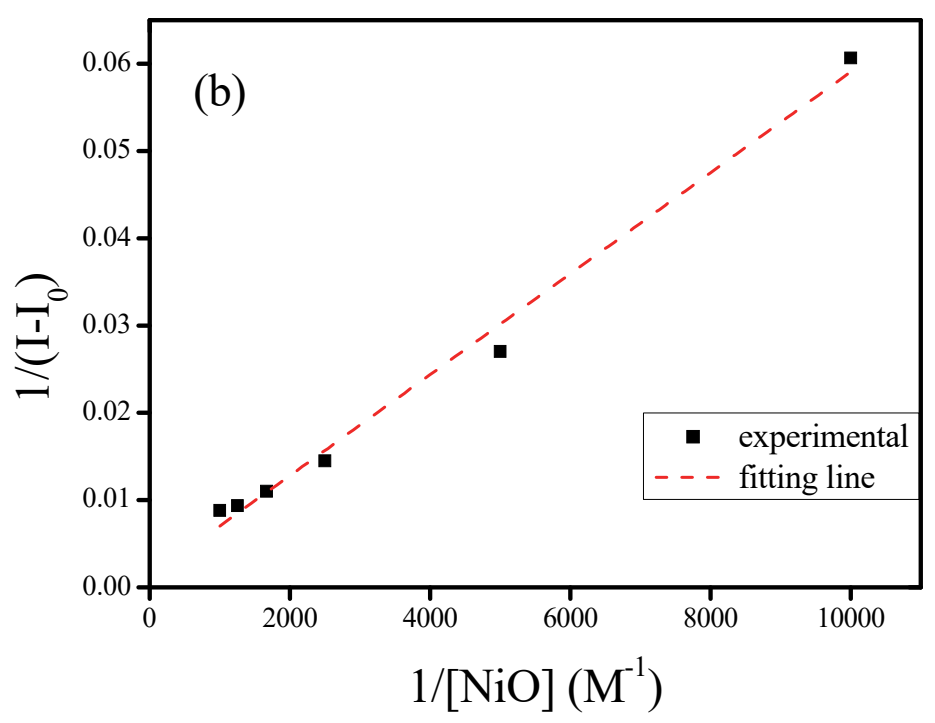

Figure 13. Double reciprocal plot for (a) glucose-NiO and (b) lactose-NiO interactions

\section{Conclusion}

$\mathrm{NiO}$ nanoparticles were successfully synthesized by co-precipitation method. They exhibited a ferromagnetic behavior that fostered their use in biosensors. The interactions of $\mathrm{NiO}$ nanoparticles with both glucose and lactose were studied by two techniques: the UV absorption and fluorescence spectroscopies. Accordingly, the binding constant was calculated from the two spectra for both glucose and lactose for further confirmation. It was found that the binding constant of $\mathrm{NiO}$ nanoparticles with lactose is slightly greater than that with glucose. This implies that $\mathrm{NiO}$ nanoparticles are candidates for glucose and lactose biosensors. Such findings postulate the utilization of $\mathrm{NiO}$ nanoparticles in lactose non-enzymatic biosensors. This study may trigger further interests and studies on metal oxide-non-enzymatic biosensors.

\section{Acknowledgments}

This work was held in the Specialized Materials Science Lab, Department of Physics and the Analytical Chemistry Lab, Department of Chemistry, Faculty of Science, Beirut Arab University. Special thanks for Professor Ashraf Moustafa Abdel-Gaber for his help throughout this work.

\section{References}

Al Boukhary, J., Zeidan, L., Khalaf, A., \& Awad, R. (2019). Synthesis, characterization, optical and magnetic properties of pure and $\mathrm{Mn}, \mathrm{Fe}$ and $\mathrm{Zn}$ dopped $\mathrm{NiO}$ nanoparticles. Journal of Chemical Physics, 516, 116-124. https://doi.org/10.1016/j.chemphys.2018.07.046

Ang, L. F., Por, L. Y., \& Yam, M. F. (2015). Development of an amperometric-based glucose biosensor to measure the glucose content of fruit. PLoS ONE, 10, e0111859. https://doi.org/10.1371/journal.pone.0111859

Barragan, C. A. R., Fornue, E. D., Ortega, J. A. A., Gonzalez, G. T., \& Quinones, H. J. C. (2016). Spectrofluorometric study of the inclusion complexation of fluorescent whitening agents and $\beta$-cyclodextrins. International Journal of Organic Chemistry, 6(4), 192-206. https://doi.org/10.4236/ijoc.2016.64020

Bian, L. J., Wang, L., Ye, Y. J., \& Liu, X. X. (2017). Glucose Biosensor Based on Immobilization Of Glucose Oxidaes On Porous Screen Printed Electrodes. International Journal of Electrochemical Sciences, 12, 62016210. https://doi.org/10.20964/2017.07.23.

Colombo, M., Carregal-Romero, S., Casula, M. F., Gutierez, L., Morales, M. P., Bohm, I. B., ... Parak, W. J. (2012). Biological applications of magnetic nanoparticles. Chemical Society Reviews, 41(11), 4306-4334. https://doi.org/10.1039/C2CS15337H

El-Kemary, M., Nagy, N., \& El-Mehasseb, I. (2013). Nickel oxide nanoparticles: Synthesis and spectral studies of interactions with glucose. Journal of Material Science in Semiconductor Processing, 16(6), 1747-1752. https://doi.org/10.1016/j.mssp.2013.05.018

Farhat, S., Rekaby, M., \& Awad, R. (2018). Synthesis and characterization of Er-doped nano ZnO samples. Journal 
of Superconductors Novel Magnetization, 31(9), 3051-3061. https://doi.org/10.1007/s10948-017-4548-9

Ferreira, L., Souza Jr, M., Trierweiler, J., Hitzmann, B., \& Foll, R. O. M. (2003). Analysis of experimental biosensor/FIA lactose measurements. Journal of Chemical Engineer, 20(1), 8. https://doi.org/10.1590/S010466322003000100003

Gao, Q., Zeng, W., \& Miao, R. (2016). Synthesis of multifarious hierarchical flower-like NiO and their gas-sensing properties. Journal of Materials Science: Materials Electron, 27(9), 9410-9416. https://doi.org/10.1007/s10854-016-4986-3

Gao, D., Zhang, Z., Fu, J., Xu, Y., Qi, J., \& Xue, D. (2009). Room temperature ferromagnetism of pure zno nanoparticles. Journal of Applied Physics, 105, 113928(1-4). https://doi.org/10.1063/1.3143103.

Hassanien, A., \& Akl, A. (2016). Effect of Se addition on optical and electrical properties of chalcogenide CdSSe thin films. Superlattices Microstructures, 89, 153-169. https://doi.org/10.1016/j.spmi.2015.10.044

Hazra, P., Chakrabarty, D., Chakraborty, A., \& Sarkar, N. (2004). Intramolecular charge transfer and solvation dynamics of Nile Red in the nanocavity of cyclodextrins. Chemical Physics Letters, 388(1-3), 150-157. https://doi.org/10.1016/j.cplett.2004.02.078

He, G., Tian, L., Cai, Y., Wu, S., Yan, H., Pu, W., ... Li, L. (2018). Sensitive nonenzymatic electrochemical glucose detection based on hollow porous NiO. Nanoscale Research Letters, 13(3), 10. https://doi.org/10.1186/s11671-017-2406-0

Jana, B., Ghosh, S., \& Chattopadhyay, N. (2011). Competitive binding of nile red between lipids and $\beta$ cyclodextrin. Journal of Photochemistry and Photobiology B: Biology, 126, 1-10. https://doi.org/10.1016/j.jphotobiol.2013.06.005

Kashani-Motlagh, M., Rahimi, R., \& Kachousangi, M. (2009). Ultrasonic method for the preparation of organic porphyrin nanoparticles. Molecules, 15(1), 280-287. https://doi.org/10.3390/molecules15010280

Khademolhoseini, S., \& Talebi, R. (2015). Green synthesis and characterization of cobalt aluminate nanoparticles and its photocatalyst application. Journal of Materials Science: Materials in Electronics, 27(3), $2938-2943$. https://doi.org/10.1007/s10854-015-4113-x

Khouri, S. J., \& Buss, V. (2011). Interaction of cationic cyanine dye with global alginates: evidence for a polymer bound dye dimer. Journal of Biophysical Chemistry, 2(4), 380-385. https://doi.org/10.4236/jbpc.2011.24043

Koh, I., \& Josephson, L. (2009). Magnetic nanoparticle sensors. Journal of Sensors, 9(10), 8130-8145. https://doi.org/10.3390/s91008130

Kolhatkar, A., Jamison, A., Litvinov, D., Willson, R., \& Lee, T. (2013). Tuning the magnetic properties of nanoparticles. International Journal of Molecular Sciences, 14(8), 15977-16009. https://doi.org/10.3390/ijms140815977

Li, S.J., Xing, Y., Hou, L., Feng, Z., Tian, Y., \& Du, J. (2016). Facile synthesis of NiO/CuO/reduced graphene oxide nanocomposites for use in enzyme-free glucose sensing. International Journal of Electrochemical Sciences, 11, 6747-6760. https://doi.org/1020964/2016.08.01

Lovic, J., Stevanovic, S., Andjelkovic, B., Petrovic, S., Vukovic, D., Prlainovic, N., ... Ivic, M. A. (2018). Electrochemical Glucose Biosensor with The Characterization Of Surface Morphology and Content Of Glucose Oxidase-Glutaraldehyde-Cysteine Layers On Gold Electrode. International Journal of Electrochemical Sciences, 13, 12340-12348. https://doi.org/10.20964/2018.12.59

Manzoori, J., \& Amjadi, M. (2003). Spectrofluorimetric study of host-guest complexation of ibuprofen with $\beta$ cyclodextrin and its analytical application. Spectrochimica Acta Part A: Molecular and Biomolecular Spectroscopy, 59(5), 909-916. https://doi.org/10.1016/S1386-1425(02)00295-0.

Meng, F., Shi, W., Sun, Y., Zhu, X., Wu, G., Ruan, C., Liu, X., \& Ge, D. (2013). Nonenzymatic biosensor based on $\mathrm{CuxO}$ nanoparticles deposited on polypyrole nanowires for improving detection range. Journal of Biosensors and Bioelectronics, 42, 141-147. https://doi.org/10.1016/j.bios.2012.10.051.

Nia, P., Meng, W., Lorestani, F., Mahmoudian, M. R., \& Alias, Y. (2015). Electrodeposition of copper oxide/polypyrrole/reduced graphene oxide as a nonenzymatic glucose biosensor. Journal of Sensors and Actuators B: Chemical, 209, 100-108. https://doi.org/10.1016/j.snb.2014.11.072.

Nur, S., Yazid, A. M., Md Isa, I., Abu Bakar, S., Hashim, N., \& Ab Ghani, S. (2014). A review of glucose biosensors based on graphene/metal oxide nanomaterials. Analytical Letters, 47(11), 1821-1834. 
https://doi.org/10.1080/00032719.2014.888731

Portaccio, M., \& Lepore, M. (2017). Determination of different saccharides concentration by means of a multienzymes amperometric biosensor. Journal of Sensors, 2017, 8. https://doi.org/10.1155/2017/7498945

Qiao, H., Wei, Z., Yang, H., Zhu, L., \& Yan, X. (2009). Preparation and characterization of NiO nanoparticles by anodic arc plasma method. Journal of Nanomaterials, 2009, 5. https://dx.doi.org/10.1155/2009/795928.

Rahal, H. T., Awad, R., Abdel Gaber, A. M., \& Bakeer, D. (2017). Synthesis, characterization, and magnetic properties of pure and edta-capped $\mathrm{NiO}$ nanosized particles. Journal of Nanomaterials, 2017, 9. https://doi.org/10.1155/2017/7460323

Rifaya, M. N., Theivasanthi, T., \& Alagar, M. (2012). Chemical capping synthesis of nickel oxide nanoparticles and their characterizations studies. Nanoscience and Nanotechnology, 2(2), 134-138. https://doi.org/10.5923/j.nn.20120205.01

Sharma, S., Kumar, A., Chaudhary, R., Suman, C., Pundir, S., \& Sehgal, N. (2007). Lactose biosensor based on lactose and galactose oxidase immobilized in polyvinyl formal. Artificial Cells, Blood Substitutes, and Biotechnology, 35(4), 421-430. https://doi.org/10.1080/10731190701460309

Sundaresan, A. \& Rao, C. (2009). Implications and consequences of ferromagnetism universally exhibited by inorganic nanoparticles. Solid State Communications, 149, 1197-1208.

Waseda, Y., Matsubara, E., \& Shinoda, K. (2011). X-Ray Diffraction Crystallography. Verlag, Berlin Heidelberg: Springer. https://doi.org/10.1007/978-3-642-16635-8

Yang, C., Liu, L., Mu, T., \& Guo, Q. (2000). The performance of the benesi-hildebrand method in measuring the binding constants of the cyclodextrin complexation. Analytical Sciences, 16(5), 537-539. https://doi.org/10.2116/analsci.16.537

Yang, H., Gong, C., Miao, L., \& Xu, F. (2017). A Glucose Biosensor Based On Horseradish Peroxidase And Glucose Oxidase Co-Entrapped In Carbon Nanotubes Modified Electrode. International Journal of Electrochemical Sciences, 12, 4958-4969. https://doi.org/10.20964/2017.06.05

Zhang, W., Chen, J., Jiang, L., Xiang, Y., \& Zhang, J. (2010). A highly sensitive nonenzymatic glucose sensor based on NiO-modified multi-walled carbon nanotubes. Journal of Microchim Acta, 168(3-4), 259-265. https://doi.org/10.1007/s00604-010-0288-2

\section{Copyrights}

Copyright for this article is retained by the author(s), with first publication rights granted to the journal.

This is an open-access article distributed under the terms and conditions of the Creative Commons Attribution license (http://creativecommons.org/licenses/by/4.0/). 